\title{
Télescope
}

Revue d'analyse comparée en administration publique

\section{The multi-level and polycentric european union: Legal and political studies, Sous la direction de Robert Grzeszczak et Ireneusz Pawel Karolewski, Berlin, Lit Verlag, 2012, 174 p.}

\section{Jean-Sébastien Marchand}

Volume 19, numéro 1, hiver 2013

URI : https://id.erudit.org/iderudit/1017160ar

DOI : https://doi.org/10.7202/1017160ar

Aller au sommaire du numéro

Éditeur(s)

L'Observatoire de l'administration publique

ISSN

1929-3348 (numérique)

Découvrir la revue

Citer ce compte rendu

Marchand, J.-S. (2013). Compte rendu de [The multi-level and polycentric european union: Legal and political studies, Sous la direction de Robert Grzeszczak et Ireneusz Pawel Karolewski, Berlin, Lit Verlag, 2012, 174 p.] Télescope, 19(1), 214-216. https://doi.org/10.7202/1017160ar d'utilisation que vous pouvez consulter en ligne. 


\section{Recensions}

\section{THE MULTI-LEVEL AND POLYCENTRIC EUROPEAN UNION: LEGAL AND POLITICAL STUDIES}

Sous la direction de Robert Grzeszczak et Ireneusz Pawel Karolewski, Berlin, Lit Verlag, 2012, $174 \mathrm{p}$.

L ouvrage The Multi-Level and Polycentric European Union: Legal and Political Studies est le fruit des travaux issus d'un séminaire ayant eu pour thème le système multiniveau et polycentrique de l'Union européenne et ayant pris place en 2010 au Centre Willy Brandt à Wrocław, en Pologne. Écrit sous la direction de Robert Grzeszczak, professeur de droit à l'Université de Varsovie, et d'Ireneusz Pawel Karolewski, professeur de science politique à l'Université de Wrocław, ce collectif cherche à explorer les aspects essentiels au thème principal, d'un angle politique et légal. Il est divisé en huit chapitres.

Le premier chapitre, coécrit par Grzeszczak et Karolewski, situe l'état de la recherche politique et légale sur le processus d'intégration de l'Union européenne (UE) et clarifie les concepts de gouvernance multiniveau (GMN) et de polycentrisme européen. Il divise aussi sommairement la suite du livre.

Dans un deuxième chapitre, Renata Duda explore l'approche " classique " des études de l'intégration européenne. Elle soutient que cette approche, dérivée des théories des relations internationales, comprend l'approche néofonctionaliste, issue de l'approche pluralistique, et l'approche intergouvernementaliste, ancrée dans la tradition néoréaliste. Toutefois, l'auteure prétend que cette approche classique explique essentiellement le processus d'intégration. Pour comprendre les aspects internes de l'intégration européenne, plus particulièrement la prise de décision et les structures de gouvernance, un nouveau concept, celui de la GMN, a donc été développé à partir d'un " amalgame de théories existantes ». Mettant la table au reste du livre, Duda souligne que la GMN est le résultat de l'observation d'une différence majeure entre les réalités internationales habituelles et l'UE, où il n'y a pas de hiérarchie ou de pouvoir unique, mais la souveraineté des États, pour lesquels la liberté est réduite par le processus transnational.

Ireneusz Pawel Karolewski poursuit avec un chapitre en deux temps : il s'attarde d'abord à la légitimité des systèmes politiques et aux caractéristiques du système multiniveau, puis il entame une réflexion sur les conséquences possibles de l'agrandissement de l'UE avec les pays de l'Est. Il conclut que l'augmentation de l'hétérogénéité, de la pluralité et de la complexité qui résulte de cet agrandissement trouve son expression dans la GMN, bien que cette même GMN exacerbe le problème de contrôle démocratique, de transparence des décisions et de responsabilité politique. 
Rafal Riedel emboîte le pas avec une étude empirique. En utilisant un modèle analytique dérivé de la GMN, il cherche à clarifier la façon dont les bureaux administratifs régionaux de la Pologne remplissent leurs fonctions depuis l'intégration du pays à l'UE. En effectuant principalement une analyse des documents administratifs et des entrevues semi-dirigées avec le personnel, il conclut que les bureaux administratifs régionaux n'utilisent pas suffisamment les possibilités offertes par le niveau supranational. Les raisons seraient à la fois les capacités organisationnelles limitées et la culture organisationnelle.

C'est ensuite au tour de Robert Grzeszczak de s'intéresser au pouvoir de l'UE, d'un point de vue légal. L'auteur met d'abord de l'ordre dans les différentes structures : le Conseil de l'Europe représente le pouvoir exécutif, le Parlement européen représente le pouvoir législatif et la Commission européenne comprend une partie de ces deux pouvoirs. Grzeszczak décortique ensuite le pouvoir exécutif de l'UE, en analysant notamment la dispersion, la balance institutionnelle, la séparation des pouvoirs, les réseaux administratifs, la légitimation et la reddition de comptes des instances exécutives.

Toujours dans une perspective légale, Agnieszka Frąckowiak-Adamska dresse, dans le chapitre suivant, un inventaire des différents régimes légaux de protections des droits fondamentaux à l'UE (standards nationaux et de l'UE, traité sur l'UE, Charte des droits fondamentaux de l'UE, conventions internationales, lois internationales et de l'UE, constitutions nationales, tradition jurisprudentielle, etc.). En explorant les interactions présentes entre les composantes de ce labyrinthe légal, elle soutient que le tout coexiste sans grands heurts, principalement en raison d'un dialogue judiciaire basé sur le respect mutuel. La communauté normative de l'UE et la Convention européenne des droits de l'homme seraient des agents facilitateurs.

L'avant-dernier chapitre, signé par Aleksandra Wentkowska, s'attarde à un problème fondamental du système légal de l'UE, c'est-à-dire la notion de bien commun. L'auteure explore d'abord cette notion sur les plans philosophique, légal, politique et sociologique. Elle examine ensuite l'introduction du concept dans la jurisprudence européenne pour comprendre comment le bien commun peut être réalisé dans l'UE. Wentkowska souligne un début de différenciation entre les notions de bien commun, d'intérêt public et d'intérêt général à la Cour de justice de l'UE, qui tend à interpréter ces notions de façon contextuelle et pragmatique.

Le dernier chapitre, écrit par Alicja Chytla, porte sur l'Initiative citoyenne européenne, ce dispositif adopté en 2007 sous le traité de Lisbonne donnant un droit d'initiative politique à une proposition venant d'au moins un million de citoyens provenant d'au moins quatre pays. L'auteure explore les possibilités et les limites des clauses de l'Initiative, conçue pour réduire le " déficit citoyen " d'une UE dirigée par des élites et un mécanisme bureaucratique complexe. Chytla soutient que ce système, malgré ses limites, contribue à réduire la perception du déficit citoyen et pourrait potentiellement contribuer à renforcer l'influence réelle des citoyens à l'échelle supranationale.

Enfin, le lecteur trouve dans ce collectif une cohérence inhabituelle pour ce type d'ouvrage. D'une part, le thème principal, développé d'abord sur le plan politique, ensuite sur le plan légal, est traité par des chercheurs, experts du sujet, 
qui partagent un regard d'universitaires d'Europe de l'Est. Cette vision des pays intégrés plus récemment à l'Union européenne apporte un vent frais aux études plus classiques sur le sujet. D'autre part, les contributions sur des angles d'approche pourtant variés demeurent étonnamment complémentaires dans l'ensemble, tant sur le plan thématique que méthodologique. Cette diversité permet un tour d'horizon rapide, sans être superficiel, des enjeux actuels du sujet.

Cependant, sans tomber dans la redondance, le lecteur aurait pu bénéficier d'une conclusion au collectif. Si le fil conducteur de l'ouvrage est relativement naturel, il n'en demeure pas moins que le lecteur privé de remarques finales est laissé à lui-même pour dénouer les différentes contributions et trouve difficilement à dire sur un apport central significatif à l'ensemble de l'œuvre. Somme toute, le résultat final demeure convaincant pour un chercheur intéressé à la gouvernance multiniveau de l'Union européenne, du point de vue politique et légal.

Par JEAN-SÉBASTIEN MARCHAND

Doctorant, École nationale d'administration publique

\section{LES DÉFIS DE LA GOUVERNANCE MULTI-NIVEAUX : EXEMPLES DU CANADA ET DE LA SUISSE}

Sous la direction de Jean-François Savard et Jean-Patrick Villeneuve, Presses de I'Université du Québec, 2011, 128 p.

C et ouvrage collectif constitue, les deux directeurs le précisent, une invitation à un questionnement encore peu exploré sur le thème du fédéralisme : celui des défis et des relations entre des administrations publiques dans les États à plusieurs paliers. La réflexion rapportée dans ce recueil est celle de cinq doctorants provenant de l'École nationale d'administration publique au Québec et de l'Institut de hautes études en administration publique de Lausanne en Suisse. À l'initiative des directeurs de l'ouvrage, ils proposent chacun un texte de réflexion autour des trois thèmes imposés par le questionnement principal : la gouvernance locale, les enjeux régionaux, les perspectives internationales.

L'ouvrage divisé en trois parties suit le plan qui a guidé la réflexion, avec un préambule introductif dans lequel les directeurs posent le cadre méthodologique et conceptuel de la démarche des doctorants.

Le premier bloc thématique, la gouvernance locale, est traité dans un premier chapitre rédigé par Caroline Jacot-Descombes sous le titre Réformes de répartition des tâches cantons-communes en Suisse : des tâches à la carte pour les communes? Cette contribution amène la question de la tension qui existe entre la décentralisation des tâches prônée par la réforme fiscale de 1999 et la centralisation que tend à dicter le principe de subsidiarité inscrit à la Constitution fédérale suisse. L'auteure conclut, après l'analyse de 13 réformes cantonales, soit 185 transferts de compétences, que 